\title{
Gallot-Tanno Theorem for closed incomplete pseudo-Riemannian manifolds and applications
}

\author{
Vladimir S. Matveev* and Pierre Mounoud
}

\begin{abstract}
We extend the Gallot-Tanno Theorem to closed pseudo-Riemannian manifolds. It is done by showing that if the cone over a manifold admits a parallel symmetric $(0,2)$-tensor then it is Riemannian. Applications of this result to the existence of metrics with distinct LeviCivita connections but having the same unparametrized geodesics and to the projective Obata conjecture are given. We also apply our result to show that the holonomy group of a closed $\left(O(p+1, q), S^{p, q}\right)$-manifold does not preserve any nondegenerate splitting of $\mathbb{R}^{p+1, q}$.
\end{abstract}

\section{Introduction.}

\subsection{Main result}

Let $(M, g)$ be a pseudo-Riemannian manifold and let $D$ be the corresponding covariant derivative. Along this paper we will consider Riemannian metrics as pseudo-Riemannian. We are interested in the existence of non-constant functions $\alpha$ on $M$ such that for all vector fields $X, Y, Z$ on $M$ we have:

$$
D D D \alpha(X, Y, Z)+c \cdot(2(D \alpha \otimes g)(X, Y, Z)+(D \alpha \otimes g)(Y, X, Z)+(D \alpha \otimes g)(Z, X, Y))=0,
$$

where $c$ is a real constant. This is a linear system of PDE on $\alpha$, in the "tensor" notation it reads

$$
\alpha_{, i j k}=c \cdot\left(2 \alpha_{, k} g_{i j}+\alpha_{, j} g_{k i}+\alpha_{, i} g_{k j}\right) .
$$

The main result of this article is the following

Theorem 1. Let $(M, g)$ be a closed (i.e., compact without boundary) connected pseudo-Riemannian manifold and $c$ be a real number. If $\alpha: M \rightarrow \mathbb{R}$ is a non-constant function satisfying (1), then $c \neq 0$ and the metric $c \cdot g$ is a Riemannian (i.e., positively definite) metric of constant curvature 1.

\subsection{History, motivation, and applications}

The equation (11) has already been studied, mostly in the Riemannian setting. The motivation of Gallot and Tanno to study this equation came from the spectral geometry: it is well-known (see for example [3]) that, on the standard sphere $S^{n} \subset \mathbb{R}^{n+1}$ of dimension $n>1$, all eigenfunctions corresponding to the second biggest eigenvalue $-n$ of the laplacian satisfy the equation

$$
D D \alpha(X, Y)+\alpha \cdot g(X, Y)=0,
$$

*partially supported by DFG (SPP 1154 and GK 1523) 
(for all vector fields $X, Y, Z$ on $M$ ). The eigenfunctions corresponding to the third biggest eigenvalue $-2(n+1)$ satisfy (1) with $c=1$.

Moreover, Obata has shown [15, Theorem A] that, on closed Riemannian manifolds, the existence of a nonconstant solution of (2) implies that the metric has constant curvature 1. Later, he [14, and, according to Gallot [3], Lichnerowicz, asked the question whether the same holds for the equation (11) (assuming $c=1$ ). The affirmative answer was given in [3, 18]. Theorem 1, which is the main result of our paper, generalizes the results of [3, 18] to pseudo-Riemannian metrics.

This equation also appears in the context of geodesic equivalence. Recall that two metrics $g$ and $g^{\prime}$ are geodesically equivalent (affinely equivalent, resp.) if every $g$-geodesic is a reparametrized $g^{\prime}$-geodesic (if their Levi-Civita connections coincide, resp.) A vector field $V$ on $(M, g)$ is called projective (affine, resp.), if its local flow acts by projective transformations (affine transformations, resp.), i.e., takes unparameterized geodesics to geodesics (preserves the Levi-Civita connection, resp.).

In particular, Solodovnikov [17] has showed that if a Riemannian metrics $g$ admits "lots" (we formalize this notion in section (5) of geodesically equivalent, but not affinely equivalent metrics, then there exists a real number $c$ and a non-constant solution of (1), see section 5 for definitions and more precise statements. This result has been recently extended to the pseudo-Riemannian setting in Kiosak et al [11]. Hiramatu [5] has shown that if a Riemannian metric of constant scalar curvature on a closed manifold admits a nonaffine projective vector field, then there also exists a nonconstant solution of the equation (11) for a certain constant $c$. Kiosak et al [7] has shown that if an Einstein metric $g$ admits a geodesically equivalent, but not affinely equivalent metric $g^{\prime}$, then there exists a non-constant solution of (11) for a certain constant $c$.

The equation (11) naturally appears in the study of the geometry of the metric cones, see Gallot [3] or Alekseevsky et al [1]. We will explain the relation between cones with decomposable holonomy and the equation (1) in section 3. In fact, this relation is one of the main tools of our proof.

Combining theorem 1 with the results listed above, we obtain that on a closed manifold any pseudo-Riemannian metric admitting "lots" of geodesically equivalent, but not affinely equivalent metrics is, up to multiplication by a constant, the Riemannian metric of constant curvature 1 (cf. corollary 5.2 ).

We also obtain that Einstein pseudo-Riemannian metrics of nonconstant curvature on closed manifolds are geodesically rigid, in the sense that every metric geodesically equivalent to them are actually affinely equivalent to them (cf. corollary 5.1).

We also obtain that metric cones with decomposable holonomy group over closed pseudoRiemannian metrics are Riemannian and flat (cf. proposition 4.1). We apply this result to closed nonzero constant curvature (non-Riemannian) manifolds i.e., to manifolds locally modeled on a pseudosphere $S^{p, q}$. We obtain that, for such a manifold, the holonomy group of the associated $\left(O(p+1, q), S^{p, q}\right)$-structure does not preserve any non degenerate splitting of $\mathbb{R}^{p+1, q}$ (cf. corollary 6.11). In [21], Zeghib proved this statement under the additional condition of completeness.

\subsection{Previous results}

Partial versions of theorem 1 were known before. In the Riemannian case, theorem 1 is due to Gallot [3, Corollaire 3.3] and Tanno [18, Theorem A] under the assumption $c>0$, and is due to Hiramatu [5, Lemma 2] under the assumption $c \leq 0$.

Moreover, Gallot and Tanno assumed only completeness (instead of closedness). In the realm of Riemannian geometry, the unit tangent bundle of a compact manifold being compact, closeness implies completeness. This is no more true in the pseudo-Riemannian geometry, where incomplete metrics on compact manifolds are abundant. For example, by Carrière et al [2] the set of 
incomplete Lorentzian 2 dimensional tori is dense in the set of Lorentzian tori. Completeness and closeness are quite independent properties in the pseudo-Riemannian geometry, and it is not an easy task to understand whether a given metric on a closed manifold is complete. Moreover, [1, Example 3.1] from Alekseevsky et al provides non-compact complete pseudo-Riemannian manifolds of non-constant curvature admitting non-constant solutions to (11). Moreover, under the additional assumption that the metric is complete theorem 1 is easy, see [12, Theorems 1,2].

\subsection{Organisation of the paper and the converse statement.}

The round sphere $S^{n}:=\left\{\left(x^{1}, \ldots, x^{n+1}\right) \in \mathbb{R}^{n+1} \mid\left(x^{1}\right)^{2}+\ldots+\left(x^{n+1}\right)^{2}=1\right\}$ with the standard metric admits a lot of nonconstant solutions of (1) (with $c=1$ ): as we mentioned in $\$ 1.2$, every eigenfunction of the Laplacian corresponding to the third biggest eigenvalue $-2(n+1)$ satisfies (1). By our theorem, any closed manifold admitting a non constant solution of (1) is, up to a constant, a quotient of $S^{n}$, but certain of those quotients do not admit nonconstant solutions of (1).

Indeed, let $M$ be the quotient of $S^{n}$ by a discrete subgroup $\Gamma \subset O(n+1)$. The cone over $M$ is the quotient of the cone over $S^{n}$ (i.e., of $\mathbb{R}^{n+1} \backslash\{0\}$ endowed with the euclidean metric) by $\Gamma$. By proposition 3.4, $M$ admits a non-trivial solution of (1), if and only if its cone is decomposable i.e., if and only if $\Gamma$ preserves an orthogonal splitting of $\mathbb{R}^{n+1}$, or equivalently if and only if there exists $0<p<n+1$ such that $\Gamma \subset O(p) \times O(n+1-p)$. Thus, the only quotients of the sphere $S^{3}$ admitting nontrivial solutions of (10) are the lens spaces. It follows that the Poincaré homology sphere (which is the quotient of the standard 3-sphere by the lift of the group of direct isometries of the regular dodecahedron) admits no nonconstant solution of (11).

The organization of the article is as follows. In section 2 we prove theorem 1 under the additional assumption $c=0$. The rest of the paper is devoted to the case $c \neq 0$ - we will explain in remark 2.1. that if $c \neq 0$, then without loss of generality we can assume $c=1$. In section 3 we establish a link between solutions of (11) (with $c=1$ ) and parallel symmetric $(0,2)$-tensors on the cone over $(M, g)$ : we show that the existence of a non-constant solution of (11) is equivalent to that the cone is decomposable. In section 4 decomposable cones are studied and theorem 1 is proved. Section 5 is devoted to the application of theorem 1 in the theory of geodesically equivalent metrics. Section 6 is devoted to the study of the holonomy of closed manifolds with constant nonzero curvature.

\section{Proof of theorem 1 under the assumption $c=0$.}

Assume $c=0$. Equation (11) implies that the Hessian of $\alpha$ is parallel. Since the manifold is closed, $\alpha$ has a minimum and a maximum. At a minimum, the Hessian must be nonnegatively definite, and at a maximum it must be nonpositevely definite. Therefore the Hessian is null, and the gradient of $\alpha$ is parallel. But as it vanishes at the extremal points, it vanishes everywhere and $\alpha$ is constant. Theorem 1 is proved under the assumption $c=0$.

Remark 2.1. If $c \neq 0$, without loss of generality we can assume $c=1$. Indeed, if a function $\alpha$ is a solution of (11) with $c \neq 0$, then it is also a solution of the equation

$$
D D D \alpha(X, Y, Z)+\left(2\left(D \alpha \otimes g^{\prime}\right)(X, Y, Z)+\left(D \alpha \otimes g^{\prime}\right)(Y, X, Z)+\left(D \alpha \otimes g^{\prime}\right)(Z, X, Y)\right)=0
$$

for $g^{\prime}:=c \cdot g$. Since the Levi-Civita connections of $g$ and of $g^{\prime}$ coincide, the equation (3) is the equation (10) with respect to the metric $g^{\prime}$ with $c=1$. 


\section{Parallel symmetric $(0,2)$-tensors on the cone over a manifold and nonconstant solutions of (1) for $c=1$.}

Let $(M, g)$ be a pseudo-Riemannian manifold. The cone manifold over $(M, g)$ is the manifold $\widehat{M}=$ $\mathbb{R}_{>0} \times M$ endowed with the metric $\widehat{g}$ defined by $\widehat{g}=d r^{2}+r^{2} g$ (i.e., in the local coordinate system $\left(r, x^{1}, \ldots, x^{n}\right)$ on $\widehat{M}$, where $r$ is the standard coordinate on $\mathbb{R}_{>0}$, and $\left(x^{1}, \ldots, x^{n}\right)$ is a local coordinate system on $M$, the scalar product in $\widehat{g}$ of the vectors $u=u_{0} \partial_{r}+\sum_{i=1}^{n} u^{i} \partial_{x^{i}}$ and $v=v_{0} \partial_{r}+\sum_{i=1}^{n} v^{i} \partial_{x^{i}}$ is given by $\left.\widehat{g}(u, v)=u^{0} v^{0}+r^{2} \sum_{i=1}^{n} g_{i j} u^{i} v^{i}\right)$.

We will denote by $D$ the Levi-Civita connection of $g$ and by $\widehat{D}$ the Levi-Civita connection of $\widehat{g}$.

The holonomy of cones over pseudo-Riemannian is strongly related to the equation (11). This relation is given by the following proposition, which is almost contained in the proofs of [3], corollaire 3.3] (for an implication) and in [12, Corollary 1] (for the reciprocal). As we will use some lines from it, as those proofs have a non empty intersection, and for the convenience of the reader, we give its proof but it does not pretend to be new.

Proposition 3.1. Let $(M, g)$ be a pseudo-Riemannian manifold, let $c=1$. Let $(\widehat{M}, \widehat{g})$ be the cone manifold over $(M, g)$.

Then, there exists a non-constant function $\alpha: M \rightarrow \mathbb{R}$ satisfying (1), if and only if there exists a non-trivial (i.e., not proportional to $\widehat{g}$ ) symmetric parallel (i.e., the covariant derivative vanishes) $(0,2)$-tensor on $(\widehat{M}, \widehat{g})$.

More precisely if $\alpha$ is a non-constant solution of (1) then the Hessian of the function $A: \widehat{M} \rightarrow \mathbb{R}$ defined by $A(r, m)=r^{2} \alpha(m)$ is non-trivial and parallel (i.e., $\left.\widehat{D} \widehat{D} \widehat{D} A=0\right)$. Conversely if $\widehat{T}$ is a non-trivial symmetric parallel $(0,2)$-tensor on $\widehat{M}$ then $\widehat{T}\left(\partial_{r}, \partial_{r}\right)$ does not depend on $r$ and is a non-constant solution of (1). Moreover $2 \widehat{T}$ is the Hessian of the function $A$ defined by $A(r, m):=$ $r^{2} \widehat{T}_{(r, m)}\left(\partial_{r}, \partial_{r}\right)$.

In the proof of proposition 3.1, we will need the following two statements; in these statements $X, Y, Z$ will denote arbitrary vector fields on $M$. We will also denote by the same letters $X, Y, Z$ the lift of these vector fields to $\widehat{M}$.

Fact 3.2 (for example, [1, 3, 12]). The Levi-Civita connection of $\widehat{g}$ is given by

$$
\widehat{D}_{X} Y=D_{X} Y-r g(X, Y) \partial_{r}, \quad \widehat{D}_{\partial_{r}} \partial_{r}=0, \quad \widehat{D}_{\partial_{r}} X=\widehat{D}_{X} \partial_{r}=\frac{1}{r} X
$$

Proof. We take a point $(r, m) \in \widehat{M}$. Without loss of generality we can assume that $D X(m)=$ $D Y(m)=D Z(m)=0$. Using $\widehat{g}\left([X, Y], \partial_{r}\right)=0$ and $\left[\partial_{r}, X\right]=\left[\partial_{r}, Y\right]=0$, we have

$$
2 \widehat{g}\left(\widehat{D}_{X} Y, \partial_{r}\right)=-\partial_{r} \cdot \widehat{g}(X, Y)=-2 r g(X, Y) .
$$

Similarly we have that $2 \widehat{g}\left(\widehat{D}_{X} Y, Z\right)=r^{2} g\left(D_{X} Y, Z\right)$. It implies the first assertion. The two others can be shown the same way.

In the next corollary we will tautologically identify $M$ with $M_{1}:=\{1\} \times M \subset \widehat{M}$ (the point $m \in M$ will be identified with $\left.(1, m) \in M_{1}\right)$. By definition of the cone metric, $\widehat{g}_{\mid M_{1}}=g$.

Corollary $3.3([12])$. Let $\widehat{T}$ be a symmetric parallel $(0,2)$-tensor on $(\widehat{M}, \widehat{g})$. Then, $\alpha:=\widehat{T}\left(\partial_{r}, \partial_{r}\right)$ does not depend on $r$ and can be considered therefore as a function on $M$. Moreover, for every $m \in M$ and every $X, Y, Z \in T_{m} M$ we have

$$
\begin{aligned}
2 \widehat{T}\left(\partial_{r}, X\right) & =r D \alpha(X) \\
2 \widehat{T}(X, Y) & =r^{2}(2 \alpha g(X, Y)+D D \alpha(X, Y)),
\end{aligned}
$$


Moreover, $\alpha$ is constant if and only if $\widehat{T}$ is proportional to $\widehat{g}$. Moreover, for every $(1, m) \in M_{1}$ we have

$$
2 D T(X, Y, Z)=-D \alpha \otimes g(Y, X, Z)-D \alpha \otimes g(Z, X, Y),
$$

where $T$ is the restriction of the tensor $\widehat{T}$ to $\left.M_{1} \quad \stackrel{\text { tautologically }}{=} M\right)$.

Proof. Since $\widehat{T}$ is parallel, we have

$$
0=\widehat{D} \widehat{T}\left(\partial_{r}, \partial_{r}, \partial_{r}\right)=\partial_{r} \cdot \widehat{T}\left(\partial_{r}, \partial_{r}\right)-2 \widehat{T}\left(\widehat{D}_{\partial_{r}} \partial_{r}, \partial_{r}\right) \stackrel{(\underline{\underline{Q}})}{=} \partial_{r} \cdot \widehat{T}\left(\partial_{r}, \partial_{r}\right)=0
$$

Thus $\widehat{T}\left(\partial_{r}, \partial_{r}\right)$ is a function on $M$. The first statement of corollary 3.3 is proved.

Combining $\widehat{D} \widehat{T}=0$ with Fact 3.2 , we have:

$$
0=\widehat{D} \widehat{T}\left(X, \partial_{r}, \partial_{r}\right)=D \alpha(X)-2 \widehat{T}\left(\widehat{D}_{X} \partial_{r}, \partial_{r}\right) \stackrel{4}{=} D \alpha(X)-\frac{2}{r} \widehat{T}\left(X, \partial_{r}\right) .
$$

This shows (5). Similarly, using Fact 3.2 and (5), we obtain

$$
\begin{aligned}
& 0=\widehat{D} \widehat{T}\left(X, Y, \partial_{r}\right)=X . \widehat{T}\left(Y, \partial_{r}\right)-\widehat{T}\left(\widehat{D}_{X} Y, \partial_{r}\right)-\widehat{T}\left(Y, \widehat{D}_{X} \partial_{r}\right) \\
& \stackrel{(44) \text {,(5) }}{=} \frac{r}{2}(D D \alpha(X, Y)+2 g(X, Y) \alpha)-\frac{1}{r} \widehat{T}(Y, X) \text {. }
\end{aligned}
$$

This shows (6). If $\alpha=$ const, $D D \alpha=0$. Then, (5)), (6), and the definition of $\alpha$ implies $\widehat{T}=\alpha \cdot \widehat{g}$. If $\widehat{T}=$ const $\cdot g$, then $\widehat{T}\left(\partial_{r}, \partial_{r}\right)=$ const $\cdot \widehat{g}\left(\partial_{r}, \partial_{r}\right)=$ const.

Similarly, using Fact 3.2 and already proved parts of corollary 3.3 , we obtain (for every $(1, M) \in$ $\left.M_{1} \subset \widehat{M}\right)$

$$
\begin{aligned}
0=\widehat{D} \widehat{T}(X, Y, Z)= & X . \widehat{T}(Y, Z)-\widehat{T}\left(\widehat{D}_{X} Y, Z\right)-\widehat{T}\left(Y, \widehat{D}_{X} Z\right) \\
\stackrel{\underline{\underline{4}}}{=} & X . T(Y, Z)-T\left(D_{X} Y, Z\right)-T\left(Y, D_{X} Z\right)+g(X, Y) \widehat{T}\left(Z, \partial_{r}\right)+g(X, Z) \widehat{T}\left(Y, \partial_{r}\right) \\
\stackrel{\text { 点 }}{=} & D T(X, Y, Z)+\frac{1}{2}(D \alpha \otimes g(Y, X, Z)+D \alpha \otimes g(Z, X, Y))
\end{aligned}
$$

implying (7).

Proof of proposition [3.1. Equation (11) being tensorial we can suppose without loss of generality that $D X=D Y=D Z=0$. We set $\widehat{X}:=\frac{1}{r} X, \widehat{Y}:=\frac{1}{r} Y, \widehat{Z}:=\frac{1}{r} Z$. By Fact 3.2 , we have:

$$
\widehat{D}_{\partial_{r}} \partial_{r}=\widehat{D}_{\partial_{r}} \widehat{X}=\widehat{D}_{\partial_{r}} \widehat{Y}=\widehat{D}_{\partial_{r}} \widehat{Z}=0, \quad \widehat{D}_{\widehat{X}} \partial_{r}=\frac{1}{r} \widehat{X} \quad \text { and } \quad \widehat{D}_{\widehat{X}} \widehat{Y}=-\frac{1}{r} g(X, Y) \partial_{r} .
$$

Let $\alpha$ be a solution of (11), and $A=r^{2} \alpha(m)$. Our first goal is to show that $\widehat{D} \widehat{D} \widehat{D} A=0$. We have:

$$
\widehat{D} A(\widehat{Z})=r D \alpha(Z) \text { and } \widehat{D} A\left(\partial_{r}\right)=2 r \alpha .
$$

Then,

$$
\widehat{D} \widehat{D} A\left(\widehat{Z}, \partial_{r}\right)=\widehat{D} \widehat{D} A\left(\partial_{r}, \widehat{Z}\right)=\partial_{r} \cdot(r D \alpha(Z))-\widehat{D} A\left(\widehat{D}_{\partial_{r}} \widehat{Z}\right)=D \alpha(Z),
$$

and similarly

$$
\widehat{D} \widehat{D} A\left(\partial_{r}, \partial_{r}\right)=2 \alpha
$$

Using that, $D Y(m)=0$, we get $Y . D A(Z)=D D A(Y, Z)$ and

$$
\widehat{D} \widehat{D} A(\widehat{Y}, \widehat{Z})=\widehat{Y} \cdot\left(r D_{Z} \alpha\right)+\frac{1}{r} g(Y, Z) \widehat{D} A\left(\partial_{r}\right)=D D \alpha(Y, Z)+2 g(Y, Z) \alpha .
$$


Now we can prove that $\widehat{D} \widehat{D} \widehat{D} A=0$, we will first show that $\widehat{D} \widehat{D} \widehat{D} A\left(\partial_{r}, .,.\right)=\widehat{D} \widehat{D} \widehat{D} A\left(., \partial_{r},.\right)=$ $\widehat{D} \widehat{D} \widehat{D} A\left(., ., \partial_{r}\right)=0$ :

$$
\begin{aligned}
& \widehat{D} \widehat{D} \widehat{D} A\left(\partial_{r}, \widehat{X}, \widehat{Y}\right) \quad=\quad \partial_{r} \cdot(\widehat{D} \widehat{D} A(\widehat{X}, \widehat{Y})) \\
& \stackrel{133}{=} \quad \partial_{r} \cdot(D D \alpha(Y, Z)+2 g(Y, Z))=0 \text {, } \\
& \widehat{D} \widehat{D} \widehat{D} A\left(\partial_{r}, \widehat{X}, \partial_{r}\right)=\widehat{D} \widehat{D} \widehat{D} A\left(\partial_{r}, \partial_{r}, \widehat{X}\right)=\partial_{r} \cdot\left(\widehat{D} \widehat{D} A\left(\partial_{r}, \widehat{X}\right)\right) \\
& \text { (111) } \partial_{r} \cdot(D \alpha(X))=0 \text {, } \\
& \widehat{D} \widehat{D} \widehat{D} A\left(\partial_{r}, \partial_{r}, \partial_{r}\right)=\partial_{r} \cdot\left(\widehat{D} \widehat{D} A\left(\partial_{r}, \partial_{r}\right)\right) \\
& \stackrel{(12)}{=} \quad 2 \partial_{r} .(\alpha)=0 \text {, } \\
& \widehat{D} \widehat{D} \widehat{D} A\left(\widehat{X}, \partial_{r}, \partial_{r}\right)=\quad \widehat{X} \cdot\left(\widehat{D} \widehat{D} A\left(\partial_{r}, \partial_{r}\right)\right)-2 \widehat{D} \widehat{D} A\left(\widehat{D}_{\widehat{X}} \partial_{r}, \partial_{r}\right) \\
& \text { 101, (12) } \widehat{X} \cdot(D \alpha(X))=0 \text {. } \\
& \widehat{D} \widehat{D} \widehat{D} A\left(\widehat{X}, \partial_{r}, \widehat{Y}\right) \quad=\quad \widehat{D} \widehat{D} \widehat{D} A\left(\widehat{X}, \widehat{Y}, \partial_{r}\right) \\
& \stackrel{[11,(91)}{=} \widehat{X} \cdot \widehat{Y} \cdot \alpha-\frac{1}{r} \widehat{D} \widehat{D} A(\widehat{X}, \widehat{Y})-\frac{1}{r} g(X, Y) \widehat{D} \widehat{D} A\left(\partial_{r}, \partial_{r}\right) \stackrel{[12],[13)}{=} 0
\end{aligned}
$$

The last thing to check is

$$
\begin{aligned}
\widehat{D}^{3} A(X, \widehat{Y}, \widehat{Z}) & =X \cdot(\widehat{D} \widehat{D} A(\widehat{Y}, \widehat{Z}))-\widehat{D} \widehat{D}\left(\widehat{D}_{X} \widehat{Y}, \widehat{Z}\right)-\widehat{D} \widehat{D}\left(\widehat{Y}, \widehat{D}_{X} \widehat{Z}\right) \\
& =X \cdot(D D \alpha(Y, Z)+2 g(Y, Z) \alpha)+\widehat{D} \widehat{D} A\left(\frac{1}{r} g(X, Y) \partial_{r}, \widehat{Z}\right)+\widehat{D} \widehat{D} A\left(\frac{1}{r} g(X, Z) \partial_{r}, \widehat{Y}\right) \\
& =D D D \alpha(X, Y, Z)+2 g(Y, Z) D \alpha(X)+g(X, Y) D \alpha(Z)+g(X, Z) D \alpha(Y)=0 .
\end{aligned}
$$

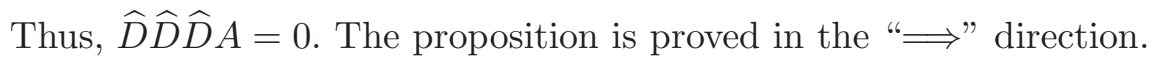

Let us now prove the proposition in the "£" direction. We take a point $(1, m) \in M_{1}$. Covariantly differentiating (6) with the help of $D$ and substituting (7), we obtain

$$
\begin{aligned}
0= & -D T(X, Y, Z)+2 g(Y, Z) X . \alpha+D D D \alpha(X, Y, Z) \\
\stackrel{(7)}{=} & 2(D \alpha \otimes g)(X, Y, Z)+(D \alpha \otimes g)(Y, X, Z)+(D \alpha \otimes g)(Z, X, Y)+D D D \alpha(X, Y, Z) .
\end{aligned}
$$

Comparing corollary 3.3 and (11), (12), (13) we see that $2 \widehat{T}$ is the Hessian of $A:=r^{2} \alpha$.

Let us recall that a pseudo-Riemannian manifold is said to be decomposable if it possess a nontrivial parallel non-degenerate (i.e., the restriction of the metric to it is nondegenerate) distribution.

By [20], if a pseudo-Riemannian manifold is decomposable, then the manifold can be locally written as the product $\left(M_{1}, g_{1}\right) \times\left(M_{2}, g_{2}\right)$ of two pseudo-Riemannian manifolds; the tangent space of $M_{1}$ naturally embedded in the tangent space of the product is precisely the parallel distribution. The tangent space of $M_{2}$ is the orthogonal complement to the parallel distribution, which is itself also a nondegenerate parallel distribution.

Contrarily to the Riemannian case, the existence of a parallel symmetric $(0,2)$-tensor on a pseudo-Riemannian manifold does not imply that the manifold is decomposable. It is a consequence of the fact that the self-adjoint endomorphism associated to such a tensor and the metric can not always be simultaneously diagonalized. However, the situation is more simple for cones over closed manifolds as shows the following

Proposition 3.4. Let $(M, g)$ be a closed pseudo-Riemannian manifold and $c=1$. If the equation (11) has a non-constant solution then $(\widehat{M}, \widehat{g})$ is decomposable. 
Proof. Let $\alpha$ be a non-constant solution of (11) on $M$. As $M$ is closed there exists two critical points $m_{-}$and $m_{+}$of $\alpha$ associated to distinct critical values (i.e., $D \alpha\left(m_{ \pm}\right)=0$ and $\alpha\left(m_{-}\right) \neq$ $\left.\alpha\left(m_{+}\right)\right)$. As $D \alpha\left(m_{ \pm}\right)=0$, it follows from (11) and (12) that, for any $r>0, \widehat{D} \widehat{D} A_{\left(r, m_{ \pm}\right)}\left(\partial_{r},.\right)$ vanishes on $T M$ and takes the value $2 \alpha\left(m_{ \pm}\right)$on $\partial_{r}$. It means that

$$
\widehat{D} \widehat{D} A_{\left(r, m_{ \pm}\right)}\left(\partial_{r}, .\right)=2 \alpha\left(m_{ \pm}\right) g_{\left(r, m_{ \pm}\right)}\left(\partial_{r}, .\right) .
$$

Since the eigenvalues of the parallel tensor are constants, $2 \alpha\left(m_{-}\right) \neq 2 \alpha\left(m_{+}\right)$are two different eigenvalues of the field of self-adjoint endomorphisms associated to $\widehat{D} \widehat{D} A$ at every points. Since the field of self-adjoint endomorphisms associated to $\widehat{D} \widehat{D} A$ is also parallel, its characteristic spaces (=generalized eigenspaces) provide a parallel orthogonal decomposition of $T \widehat{M}$. Indeed, they are clearly nondegenerate; as there are at least two distinct eigenvalues each characteristic space is non trivial. Then, $(\widehat{M}, \widehat{g})$ is decomposable.

Proposition 3.4 does not say that a cone over a closed manifold with interesting holonomy is automatically decomposable. For example, the cone may admit anti-symmetric parallel $(0,2)$-tensors. The reader can consult Alekseevsky et al [1] for a more systematic study of the holonomy of cones.

\section{Decomposable cones over closed manifolds and the proof of The- orem 1.}

The goal of this section is to prove

Proposition 4.1. Let $(M, g)$ be a closed connected pseudo-Riemannian manifold such that the cone $(\widehat{M}, \widehat{g})$ is decomposable. Then, $\widehat{g}$ is the Riemannian flat metric, and $g$ is the Riemannian metric of constant curvature 1 .

Proof. Let $V_{1}$ and $V_{2}:=V_{1}^{\perp}$ be the complementary nondegenerate parallel distribution on $\widehat{M}$. Let $\widehat{T}_{1}$ and $\widehat{T}_{2}$ be the symmetric $(0,2)$-tensors on $\widehat{M}$ defined for $i \in\{1,2\}$ by

$$
\widehat{T}_{i}(v, u)=\widehat{g}\left(v_{i}, u\right)
$$

where the $v_{i}$ 's are the factors of the decomposition of $v$ according to the splitting $T \widehat{M}=V_{1} \oplus V_{2}$. Clearly, $\widehat{T}_{1}+\widehat{T}_{2}=\widehat{g}$. Since the distributions $V_{i}$ are parallel, then the tensors $\widehat{T}_{i}$ are also parallel.

We set $\alpha_{i}:=\widehat{T}_{i}\left(\partial_{r}, \partial_{r}\right)$. Since $\widehat{g}\left(\partial_{r}, \partial_{r}\right)=1$, we have $\alpha_{1}+\alpha_{2}=\widehat{T}_{1}\left(\partial_{r}, \partial_{r}\right)+\widehat{T}_{2}\left(\partial_{r}, \partial_{r}\right)=$ $\widehat{g}\left(\partial_{r}, \partial_{r}\right)=1$. As in section [3, we define the functions $A_{1}$ and $A_{2}$ on $\widehat{M}$ by $A_{i}(r, m)=r^{2} \alpha_{i}(m)$.

Applying proposition 3.1 to the tensors $\widehat{T}_{i}$ we obtain that the following statements hold for every $i=1,2$ :

(*) $\alpha_{i}$ is a non constant function on $M$ and it is a solution of the equation (1).

(**) The Hessian of $A_{i}$ is $2 \widehat{T}_{i}$.

Let us prove that the only possible critical values of $\alpha_{i}$ are 0 and 1 . Moreover, for any $m \in M$, we have $0 \leq \alpha_{i}(m) \leq 1$, the extremal values being reached.

Since $\alpha_{1}+\alpha_{2}=1$, it is sufficient to prove this statement for $\alpha_{1}$. Let $m \in M$ be a critical point of $\alpha_{1}$. As we already saw, at (14), it implies that at the point $(r, m)$

$$
2 \alpha_{1}(m) g\left(\partial_{r}, .\right)=\widehat{D} \widehat{D} A_{1}\left(\partial_{r}, .\right) \stackrel{(* *)}{=} 2 \widehat{T}_{1}\left(\partial_{r}, .\right) .
$$


Then, $\partial_{r}(r, m)$ is an eigenvector of the self-adjoint endomorphism associated to $\widehat{T}_{1}$, and $\alpha_{1}(m)$ is the eigenvalue of this endomorphism. Since the only eigenspaces of $\widehat{T}_{1}$ are $V_{1}$ (with eigenvalue 1) and $V_{2}$ (with eigenvalue 0 ), then $\alpha_{1}(m)=0$ or $\alpha_{1}(m)=1$. Thus, the only critical values of $\alpha_{1}$ are 0 and 1.

Since $M$ is closed, there exists $m_{1}, m_{0} \in M^{2}$ such that $\alpha_{1}\left(m_{1}\right)=\max _{m \in M} \alpha_{1}(m)$ and $\alpha_{1}\left(m_{0}\right)=$ $\min _{m \in M} \alpha_{1}(m)$. Then, $d \alpha_{1}\left(m_{1}\right)=d \alpha_{1}\left(m_{0}\right)=0$, implying $\alpha_{1}\left(m_{1}\right)=1$ and $\alpha_{1}\left(m_{0}\right)=0$.

Let us prove that the tensor $\widehat{T}_{1}$ is nonnegatively definite. We take the point $\left(r, m_{0}\right) \in \widehat{M}$. This point is a minimum of the function $A_{1}$. Indeed, $A_{1}(r, m)=r^{2} \alpha_{1} \geq 0$, and $A_{1}\left(r, m_{0}\right)=r^{2} \alpha_{1}\left(m_{0}\right)=$ 0 . Since $\left(r, m_{0}\right)$ is a minimum, at this point $\widehat{D} \widehat{D} A_{1}$ is nonnegatively defined. Since $2 \widehat{T}_{1} \stackrel{(* *)}{=} \widehat{D} \widehat{D} A_{1}$, $\widehat{T}_{1}$ is nonnegatively defined at the point $\left(r, m_{0}\right)$. Since $\widehat{T}_{1}$ is parallel, it is also nonnegatively defined at every point of $\widehat{M}$.

Similarly, one can prove that $\widehat{T}_{2}$ is nonnegatively defined: instead of the point $\left(r, m_{0}\right)$ one should take the point $\left(r, m_{1}\right)$ where the function $A_{2}$ accepts its minimum.

Since $\widehat{g}=\widehat{T}_{1}+\widehat{T}_{2}$, it is also nonnegatively defined. Since it is nondegenerate, it is positively defined, i.e., is a Riemannian metric. As we recalled in the introduction, the Riemannian version of theorem 1 was proved by Gallot [3] and Tanno [18. Thus, by Gallot-Tanno Theorem, $g$ has constant curvature equal to 1 , and $\widehat{g}$ is the Riemannian flat metric.

Remark 4.2. The hypothesis of compactness in Proposition 4.1 is only use to obtain that the function $\alpha_{i}$ defined during the proof has a minimum and a maximum. Hence, we could replace the hypothesis of compactness by this weaker one.

Proof of theorem 1. The case $c=0$ was done in section 2, By remark 2.1, we can assume $c=1$. By proposition 3.4, the existence of a nonconstant solution of (1) implies that the cone $(\widehat{M}, \widehat{g})$ is decomposable. By proposition 4.1, $g$ is a Riemannian metric of constant curvature 1 .

\section{Application I: geodesic rigidity of Einstein manifolds and pro- jective Obata conjecture.}

The set of metrics geodesically equivalent (the definition is in $\$ 1.2$ ) to a metric $g$ is in one-to-one correspondence with the set of nondegenerate symmetric $(0,2)$-tensors $T$ such that for any vector fields $X, Y, Z$ on $M$

$$
D T(X, Y, Z)=\frac{1}{2}(D \operatorname{trace}(T) \otimes g(Y, X, Z)+D \operatorname{trace}(T) \otimes g(Z, X, Y)),
$$

where the trace and the covariant derivative are taken according to $g$, see for example [7, §2.2] for details (in the "tensor" notations, the equation (15) reads $T_{i j, k}=\frac{1}{2}\left(T_{p, i}^{p} g_{j k}+T_{p, j}^{p} g_{i k}\right)$.)

Since this equation is linear, the space of its solutions is a linear vector space. Its dimension is called the degree of mobility of $g$.

Locally, the degree of mobility of $g$ coincides with the dimension of the set (equipped with natural topology) of metrics geodesically equivalent to $g$.

It is easy to see that if $\alpha$ is a solution of the equation (11) then the tensor defined by (13) is a solution of (15). Indeed, one can check it directly, or one can use that for the covariantly-constant by proposition 3.1 tensor $\widehat{T}=\widehat{D} \widehat{D} A$ the tensor (13) is precisely the tensor $T$ from corollary 3.3 . Then, it satisfies the equation (7), which is equivalent to (15).

In some cases the reciprocal is true, hence theorem 1 has the following corollaries. 
Corollary 5.1. Let $g$ be an Einstein (i.e., the Ricci tensor is proportional to g) pseudo-Riemannian metric on an $(n>2)$-dimensional closed connected manifold. Assume that $\bar{g}$ is geodesically equivalent to $g$, but is not affinely equivalent to $g$. Then for a certain constant $c \neq 0$ the metric $c \cdot g$ is the Riemannian metric of constant curvature 1.

Proof. By [7, Corollary 3], if the metric $g$ is Einstein and if there exists a geodesically equivalent, but not affine equivalent metric $\bar{g}$, then the equation (11) admits a non-constant solution. The corollary therefore follows from theorem 1 .

Corollary 5.2. Let $g$ be a pseudo-Riemannian metric on an $(n>1)$-dimensional closed connected manifold. Then, if the metric $\bar{g}$ on $M$ is geodesically equivalent to $g$, but not affinely equivalent to $g$, then the degree of mobility of $g$ is precisely 2 or for certain constants $c \neq 0 \neq \bar{c}$ the metrics $c \cdot g$ and $\bar{c} \cdot \bar{g}$ are Riemannian metrics of constant curvature 1.

Proof. Assume first that $n=\operatorname{dim}(M) \geq 3$. Under this assumption, by [6] if the degree of mobility of $g$ is $\geq 3$, then for every solution $T$ of (15), the function $f:=\operatorname{trace}(T)$ is a solution of (1). More precisely, [6. Lemma 3 and Corollary 4] implies that in a neighborhood of almost every point there exists a constant $c$ such that $f$ is a solution of (11). Now, by [6. Lemma 7 in $\S 2.3 .4$ ] the constant $c$ is actually universal (implying that the equation (1) is fulfilled on the whole manifold). In this case the result follows therefore from Theorem 1 .

Now, the case $n=\operatorname{dim}(M)=2$ follows from [8, Theorem 5.1] for the signatures $(+,+)$ and $(-,-)$, and from [13, Corollary 1] for the signature $(+,-)$.

Corollary 5.2 is related to the following classical conjecture:

Projective Obata conjecture. Let $G$ be a connected Lie group acting on a closed connected pseudo-Riemannian or Riemannian manifold $(M, g)$ of dimension $n>1$ by projective transformations. Then it acts by affine transformations or there exists a constant $c \neq 0$ such that $(M, c \cdot g)$ is the quotient of a Riemannian round sphere.

By corollary 5.2, we have:

Corollary 5.3. If $\left(M^{n}, g\right)$ is a counter-example to the projective Obata conjecture, then $n:=$ $\operatorname{dim}\left(M^{n}\right) \geq 3$ and the degree of mobility of $g$ is precisely 2 .

Proof. The existence of a projective nonaffine transformation for $g$ implies the existence of a metric that is geodesically equivalent to $g$, but is not affine equivalent to $g$. By corollary 5.2, if $n=\operatorname{dim}\left(M^{n}\right) \geq 3$, the degree of mobility of $g$ is 2 .

Now, by [13, Theorem 6], the projective Obata conjecture is true in dimension two.

In the Riemannian case, projective Obata conjecture was proved in [10, Theorem 1] for dimension 2 and in [11, Corollary 1] for dimensions $\geq 3$. The natural idea to prove the conjecture in the pseudo-Riemannian case is to mimic the Riemannian proof for pseudo-Riemannian metrics. The (Riemannian) proof contains two parts:

(i) proof for the metrics with the degree of mobility 2 ([11, Theorem 15]),

(ii) proof for the metrics with the degree of mobility $\geq 3$ ([11, Theorem 16]).

We expect that it is possible, though nontrivial, to generalize (i) for the pseudo-Riemannian case. On the other side, one can not expect to generalize (ii) for pseudo-Riemannian metrics, because (ii) is based on Riemannian results that are no more true in a pseudo-Riemannian setting. Hence corollary 5.3 proves the part that was, a priori, the most difficult part of the projective Obata conjecture for pseudo-Riemannian metrics. 
Moreover, the next corollary shows that the group of projective transformations of a closed manifold coincides with the group of affine transformations, or the group of isometries has codimension one in the group of projective transformations.

Corollary 5.4. Let $(M, g)$ be a closed connected $(n>1)$-dimensional pseudo-Riemannian manifold. Assume that for no constant $c \in \mathbb{R} \backslash\{0\}$ the metric $c \cdot g$ is the Riemannian metric of constant curvature 1. Then, every projective vector field is an affine vector field, or certain nontrivial linear combination of every two projective vector fields is a Killing vector field.

Proof. Indeed, it is well known (see, for example [11, or more classical sources acknowledged therein) that a vector field $X$ is projective if the tensor

$$
T:=\mathcal{L}_{X} g-\frac{1}{n+1} \operatorname{trace}\left(\mathcal{L}_{X} g\right) \cdot g
$$

is a solution of (15), where $\mathcal{L}_{X}$ is the Lie derivative with respect to $X$. Moreover, the projective vector field is affine, if and only if the trace of $T$ is constant.

Suppose the degree of mobility of $g$ is not 2. Then, corollary 5.2 implies that all projective vector fields are actually affine, which is one of the possibilities in corollary 5.4.

Now, suppose the degree of mobility of $g$ is precisely 2. Let $X$ and $Y$ be projective vector fields. We consider the solutions $T=\mathcal{L}_{X} g-\frac{1}{n+1} \operatorname{trace}\left(\mathcal{L}_{X} g\right) g$ and $T^{\prime}=\mathcal{L}_{Y} g-\frac{1}{n+1} \operatorname{trace}\left(\mathcal{L}_{Y} g\right) \cdot g$ of (15). Since the degree of mobility is $2, T, T^{\prime}$, and $g$ are linearly dependent, i.e., for certain constants $k, k^{\prime}, l$ we have $k T+k^{\prime} T^{\prime}=l g$. Since the mapping

$$
X \mapsto \mathcal{L}_{X} g-\frac{1}{n+1} \operatorname{trace}\left(\mathcal{L}_{X} g\right) g
$$

is linear, we have

$$
\mathcal{L}_{k X+k^{\prime} Y} g-\frac{1}{n+1} \operatorname{trace}\left(\mathcal{L}_{k X+k^{\prime} Y} g\right) g=l g \text { implying } \mathcal{L}_{k X+k^{\prime} Y} g=(n+1) l g,
$$

i.e., $k X+k^{\prime} Y$ is a homothety vector field (if $l \neq 0$ ) or a Killing vector field (if $l=0$ ).

Since $M$ is closed, it admits no homotheties implying $k X+k^{\prime} Y$ is a Killing vector field.

\section{Application II: Holonomy groups of closed constant curvature manifolds.}

Let $X$ be a manifold and $G$ be a Lie group acting analytically on $X$. A $(G, X)$-structure on a manifold $M$ is given by an atlas $\left(U_{i}, \varphi_{i}\right)$ such that each $\varphi_{i}$ takes values in $X$ and each transition function $\varphi_{i} \circ \varphi_{j}^{-1}$ is the restriction of the action of an element of $G$ on $X$.

If $M$ has a $(G, X)$-structure then there exists (see for example [19, pp. 140,141] for details) a local diffeomorphism $\delta: \widetilde{M} \rightarrow \widetilde{X}$, where $\widetilde{M}$ and $\widetilde{X}$ are the universal covers of $M$ and $X$ respectively, and a morphism $\rho: \pi_{1}(M) \rightarrow \widetilde{G}$ (where $\pi_{1}(M)$ denotes the fundamental group of $M$, which acts as the group of deck transformations of the covering $\widetilde{M} \rightarrow M$, and $\widetilde{G}$ denotes the covering of $G$ that acts on $\widetilde{X})$ such that, for any $\gamma \in \pi_{1}(M)$ and any $\tilde{m} \in \widetilde{M}$, we have $\delta(\gamma \cdot \tilde{m})=\rho(\gamma) . \delta(\tilde{m})$. The map $\delta$ is called the developing map and the morphism $\rho$ is called the holonomy morphism. The image of $\pi_{1}(M)$ with respect to $\rho$ is called the holonomy group of the $(G, X)$-manifold $M$, it contains a lot of informations about the geometry of $M$.

We denote by $\mathbb{R}^{p+1, q}$ the space $\mathbb{R}^{p+q+1}$ equipped with the standard pseudo-Euclidean metric of signature $(p+1, q)$, and consider the pseudo-sphere $S^{p, q}=\left\{x \in \mathbb{R}^{p+1, q} \mid\langle x, x\rangle=1\right\}$ and $O(p+$ $1, q) \ltimes \mathbb{R}^{p+q+1}$ the isometry group of $\mathbb{R}^{p+1, q}$. We recall that $S^{p, q}$ is simply connected if and only if $p \neq 1$. 
It is well known that every pseudo-Riemannian manifold of signature $(p, q)$ and constant curvature equal to 1 is a manifold with a $\left(O(p+1, q), S^{p, q}\right)$-structure and that the flat pseudo-Riemannian manifolds of signature $(p+1, q)$ are the manifolds having a $\left(O(p+1, q) \ltimes \mathbb{R}^{p+q+1}, \mathbb{R}^{p+1, q}\right)$-structure.

The holonomy groups of those $(G, X)$-manifolds are not the usual pseudo-Riemannian holonomy groups (even if they are closely related). For now on we will only consider holonomy groups of $(G, X)$-structures. Proposition 4.1 implies

Corollary 6.1. If $q \neq 0$, the action of the holonomy group of a closed manifold endowed with a $\left(O(p+1, q), S^{p, q}\right)$-structure (i.e. endowed with a pseudo-Riemannian metric with constant curvature equal to 1) on $\mathbb{R}^{p+1, q}$ does not preserve any non-degenerate splitting.

Corollary 6.1 was known under the additional assumption that the manifold is complete, see [21, Fact 2.3]. Since by [9] any constant curvature Lorentz manifold is complete, corollary 6.1 was also known for closed manifolds of Lorentz signature. If $q=0$, the sphere itself is a counterexample.

Proof of corollary 6.1. In order to simplify the notation, we will suppose $p \neq 1$, i.e., that $S^{p, q}$ is simply connected. Anyway, if $p=1, M$ is Lorentzian, therefore complete by [9] and the corollary follows from [21]. Moreover, it is easy to adapt what follows to the case $p=1$.

Let $(M, g)$ be a closed pseudo-Riemannian manifold with constant curvature equal to 1 . We denote by $\widetilde{M}$ the universal cover of $M$ and by $\widehat{\widetilde{M}}$ the cone over its universal cover. It is well-known (and follows from Fact 3.2) that the curvature of the cone metric $\widehat{g}=d r^{2}+r^{2} g$ is given by

$$
\widehat{R}(X, Y) Z=R(X, Y) Z-g(Y, Z) X+g(X, Z) Y,
$$

where $R$ and $\widehat{R}$ are the curvatures of $g$ and of $\widehat{g}$. It implies that $(\widehat{M}, \widehat{g})$ is flat.

We identify $\widehat{S^{p, q}}$, the cone over the pseudosphere $S^{p, q}$, with $\left\{x \in \mathbb{R}^{p+1, q} \mid\langle x, x\rangle>0\right\}$. Let $\delta: \widetilde{M} \rightarrow S^{p, q}$ be a developing map of the induced $\left(O(p+1, q), S^{p, q}\right)$-structure on $M$. The map $\widehat{\delta}$ : $\widehat{\widetilde{M}} \rightarrow \widehat{S^{p, q}}$ defined by $\widehat{\delta}(r, m):=(r, \delta(m))$ is a developing map of the $\left(O(p+1, q) \ltimes \mathbb{R}^{p+q+1}, \mathbb{R}^{p+1, q}\right)$ structure of the flat manifold $\widehat{M}$. The holonomy morphisms associated to $\widehat{\delta}$ and $\delta$ are clearly the same. We denote them by $\rho$.

Let $T_{0}$ be a symmetric parallel $(0,2)$ - tensor on $\mathbb{R}^{p+1, q}$ (i.e. a symmetric bilinear form) invariant with respect to the holonomy group $\rho\left(\pi_{1}(\widehat{M})\right)$. Let $\widehat{\widetilde{T}}=\widehat{\delta}^{*} T_{0}$ be the pull back of $T_{0}$ by $\widehat{\delta}$. Let $\gamma$ be an element of $\pi_{1}(\widehat{M})$ seen as the group of deck transformations of the universal covering. We have $\gamma^{*} \widehat{\widetilde{T}}=\gamma^{*}\left(\widehat{\delta}^{*} T_{0}\right)=\widehat{\delta}^{*}\left(\rho(\gamma)^{*} T_{0}\right)$, but as we supposed that $\rho(\gamma)^{*} T_{0}=T_{0}$ it implies that $\widehat{\widetilde{T}}$ is invariant by the action of $\pi_{1}(M)$. It means that $\widehat{\widetilde{T}}$ is the pull-back of a parallel tensor $\widehat{T}$ on $\widehat{M}$.

By propositions 3.1, 3.4 and 4.1, the tensor $\widehat{T}$ is proportional to the metric $\widehat{g}$. Thus $T_{0}$ also is proportional to the metric of $\mathbb{R}^{p+1, q}$. It means that the holonomy group of a closed $\left(O(p+1, q), S^{p, q}\right)$-manifold does not preserve any symmetric bilinear form on $\mathbb{R}^{p+1, q}$ which is not proportional to the metric. In particular it does not preserve any non-degenerate splitting of $\mathbb{R}^{p+1, q}$.

Note that a stronger version of corollary 6.1 exists for flat pseudo-Riemannian manifolds. More precisely, by Goldman et al [4] the holonomy of a closed affine manifold admitting a parallel volume form (for example pseudo-Riemannian and flat) does not preserve any non trivial subspace.

As the following example shows, corollary 6.1 is no more true for degenerate splittings. We identify $\mathbb{R}^{2,2}$ with $M(2, \mathbb{R}$ ) the space of order 2 square matrices endowed with the determinant (seen as a quadratic form). The pseudo-sphere is then identified with $S L(2, \mathbb{R})$. For any $v \in \mathbb{R}^{2} \backslash\{0\}$, we define the set $V_{v}$ by $V_{v}=\{M \in M(2, \mathbb{R}) ; M . v=0\}$. They are 2-dimensional totally degenerate subspaces. If $v$ and $w$ are not colinear, we have $\mathbb{R}^{2,2}=V_{v} \oplus V_{w}$. Furthermore, $S L(2, \mathbb{R})$ clearly 
acts isometrically by left multiplication on $M(2, \mathbb{R})$. This action preserves $V_{v}$ and $V_{w}$. Now, let $\Gamma$ be a cocompact lattice in $P S L(2, \mathbb{R})$. The manifold $P S L(2, \mathbb{R}) / \Gamma$ is a closed 3 dimensional anti de Sitter manifold whose holonomy lies in $S L(2, \mathbb{R})$ and therefore preserves certain totally degenerate splittings of $\mathbb{R}^{2,2}$.

However, up to the authors knowledge, these examples (and some of their deformations cf. [16]) are the only known examples of closed pseudo-Riemannian manifolds of constant curvature whose holonomy preserves a non trivial degenerate subspace. Moreover, the main proposition of [21] is actually that the holonomy group of a closed anti de Sitter manifold of dimension greater than 3 is irreducible.

Acknowledgement: V.M. thanks Deutsche Forschungsgemeinschaft (Priority Program 1154 Global Differential Geometry and research training group 1523 - Quantum and Gravitational Fields) and FSU Jena for partial financial support, and Vicente Cortes and Dmitri Alekseevsky for useful discussions.

\section{References}

[1] D.V. Alekseevsky, V. Cortes, A.S. Galaev, T. Leistner, Cones over pseudo-Riemannian manifolds and their holonomy, J. Reine Angew. Math. (Crelle's journal), Band 2009, Heft 635, 23-69, arXiv:0707.3063v2

[2] Y. Carrière, L. Rozoy, Complétude des métriques lorentziennes de $T^{2}$ et difféormorphismes du cercle, Bol. Soc. Brasil. Mat. (N.S.) 25(1994), no. 2, 223-235.

[3] S. Gallot, Équations différentielles caractéristiques de la sphère, Ann. scient. Éc. Norm. Sup. $4^{e}$ série 12(1979) 235-267.

[4] W. Goldman, M.W. Hirsch, The radiance obstruction and parallel forms on affine manifolds, Trans. AMS 286(1984), no. 2, 629-649.

[5] H. Hiramatu, Riemannian manifolds admitting a projective vector field, Kodai Math. J. 3(1980), no. 3, 397-406.

[6] V. Kiosak, V. S. Matveev, Proof of projective Lichnerowicz conjecture for pseudo-Riemannian metrics with degree of mobility greater than two, accepted to Comm. Math. Phys. arXiv:0810.0994v4.

[7] V. Kiosak, V. S. Matveev, Complete Einstein metrics are geodesically rigid, Comm. Math. Phys. 289(2009), no. 1, 383-400, arXiv:0806.3169.

[8] K. Kiyohara, Compact Liouville surfaces, J. Math. Soc. Japan 43(1991), 555-591.

[9] B. Klingler, Complétude des variétés lorentziennes à courbure constante, Math. Ann. 306 (1996), no. 2, 353-370.

[10] V. S. Matveev, Die Vermutung von Obata für Dimension 2, Arch. Math. 82(2004), 273-281.

[11] V. S. Matveev, Proof of the Lichnerowicz Conjecture, J. Diff. Geom. 75(2007), 459-502, arXiv:math/0407337 
[12] V. S. Matveev, Gallot-Tanno theorem for pseudo-Riemannian manifolds and a proof that decomposable cones over closed complete pseudo-Riemannian manifolds do not exist, J. Diff. Geom. Appl., 28(2010) no. 2, doi:10.1016/j.difgeo.2009.10.009, arXiv:0906.2410

[13] V. S. Matveev, Pseudo-Riemannian metrics on closed surfaces whose geodesic flows admit nontrivial integrals quadratic in momenta, and proof of the projective Obata conjecture for two-dimensional pseudo-Riemannian metrics, arXiv:1002.3934

[14] M. Obata, Riemannian manifolds admitting a solution of a certain system of differential equations, Proc. U.S.-Japan Seminar in Differential Geometry (Kyoto, 1965) pp. 101-114.

[15] M. Obata, Certain conditions for a Riemannian manifold to be isometric with a sphere, J. Math. Soc. Japan 14(1962), 333-340.

[16] F. Salein, Variétés anti-de Sitter de dimension 3 possédant un champ de Killing non trivial, C. R. Acad. Sci. Paris Sr. I Math. 324 (1997), no. 5, 525-530.

[17] A. S. Solodovnikov, Projective transformations of Riemannian spaces, Uspehi Mat. Nauk (N.S.) 11(1956), no. 4(70), 45-116.

[18] S. Tanno, Some differential equations on Riemannian manifolds, J. Math. Soc. Japan 30(1978), no. $3,509-531$.

[19] W. Thurston, Three dimensional Geometry and Topology, vol. 1, Princeton Mathematical Series, vol. 35, Princeton University Press, Princeton, NJ,1997, edited by Silvio Levy.

[20] H. Wu, On the de Rham decomposition theorem, Illinois J. Math. 8(1964), 291-311.

[21] A. Zeghib, On closed anti de Sitter spacetimes, Math. Ann. 310(1998), 695-716.

Vladimir S. Matveev

Address: Institute of Mathematics, FSU Jena, 07737 Jena Germany

E-mail: vladimir.matveev@uni-jena.de

Pierre Mounoud

Address: Université Bordeaux 1, Institut de Mathématiques de Bordeaux, 351, cours de la libération, F-33405 Talence, France

E-mail: pierre.mounoud@math.u-bordeaux1.fr 